\title{
In vivo quercetol effect in lead acetate poisoning
}

\author{
Florentina RONCEA*, Horatiu MIRESAN, Adrian Cosmin ROSCA, Mihaela BRATU and Antoanela \\ POPESCU
}

\author{
Ovidius University of Constanta, Faculty of Pharmacy, University Street No.1, Constanta, Romania
}

\begin{abstract}
The present study investigated the influence of quercetol upon $\delta$-aminolevulinic acid $(\Delta-$ ALA) urine concentration as marker of lead poisoning. The study was conducted on six lots of 6 mature Wistar rats of both sexes, lots not poisoned treated with different concentrations of quercetol (Q1, Q2), control (L6M), lot poisoned untreated $(\mathrm{L} 3 \mathrm{~Pb})$, lots poisoned and treated with $\mathrm{Q}(\mathrm{L} 4 \mathrm{Q} 1 \mathrm{~Pb}$ and L5Q2Pb). After 11 days urine from 24 hours was collected for $\Delta$-ALA spectrophotometric assay and testing the significance of mean difference of by " $\mathrm{t}$ " test Student at $\mathrm{p}<0.05$. Statistical analysis of the data presented shows that compared to L2Q2 and L6M the amount of $\delta$-ALA excreted in urine under quercetol influence $(\mathrm{L} 4 \mathrm{Q} 1 \mathrm{~Pb})$ shows statistical significance compared to (L2Q2) the amount of $\delta$-ALA excreted in urine compared to ( $\mathrm{L} 3 \mathrm{~Pb})$ shows statistical significance. Different concentrations of quercetol (Q1, Q2), did not produce significant changes in the $\delta$-ALA excreted compared with values of $(\mathrm{L} 3 \mathrm{~Pb})$. Difference between means is probably due to sampling fluctuation, is not significant, reduced growth to eliminate $\delta$-ALA on $\mathrm{L} 4 \mathrm{Q} 1 \mathrm{~Pb}$ and $\mathrm{L} 5 \mathrm{Q} 2 \mathrm{~Pb}$ is believed to be due to iron complex formation, reducing hemoglobin synthesis. From the results we conclude that hem biosynthesis does not start to grow under quercetol protection. The obtained data are not relevant statistical since interpretations were performed on non homogeneous groups in number of individuals, the percentage of mortality variability and high levels of standard deviation calculated from each lot.
\end{abstract}

Keywords: lead acetate poisoning, quercetol, $\delta$-aminolevulinic acid urine assay, possible antidote.

\section{Introduction}

As unprofessional poisoning with inorganic lead compounds produce haematotoxicity (enzyme inhibition with blocking chain thiolofore porphyrin and hem biosynthesis interference, increased $\delta$ aminolevulinic acid in serum, urine elimination increased, decreased hemoglobin and number of erythrocytes, erythrocyte membrane alteration) and nephrotoxicity (shown to tubules convoluted proximal and distal) and antidotes used show nonspecificity and toxicity (nephrotoxicity, depletion $\mathrm{Ca}^{2+}, \mathrm{Zn}^{2+}$ ions effects on skin) [1 - 7] we chosed chelating - natural ligands (quercetol, rutoside) that have fewer toxic effects and act as an possible antidote.

One mechanism involved in lead poisoning is aerobic oxidation of $\delta$-aminolevulinic acid, metal catalyzed reaction at physiological $\mathrm{pH}$, with appearance of several species of free radicals, which cause oxidative stress at the level of the accumulated $\delta$-aminolevulinic acid (kidney) or autooxidation and enolisation [8 - 10].

The consequence consists in increased activity of stress enzymes, superoxide dismutase and catalase, due to alteration of cell membrane and hemolysis caused by lead $[12,13]$.

Quercetol (3, 3', 4', 5 ,7-pentahidroxiflavone) selection as a vegetal principle with possible antidote properties is based on multiple experimental observations: it forms stable complexes with plurivalent metal ions $\left(\mathrm{Cu}^{2+}, \mathrm{Fe}^{2+}, \mathrm{Fe}^{3+}, \mathrm{Zn}^{2+}\right.$, $\mathrm{Al}^{+3}$ ) [1], in vitro verification of these complexes formation and stability [2 - 4], in vivo decrease of oxidative stress (superoxiddismutase, catalase) in crustaceans (Euxinia maeotica) and molluscs (Mytillus galloprovincialis) poisoned with lead 
acetate $[5,7]$, annihilation of some toxic lead acetate poisoning effects $[8,9]$, free radicals captation (inhibition of membrane lipids peroxidation, protection against oxidative stress - antioxidant activity), and even neuroprotective effect $[10,11]$, when the plasmatic concentration of homovanilic acid increases [10 - 12].

Previously conducted researches on mice poisoned with lead acetate and treated with quercetol revealed quercetol role in lowering enzymatic activity of superoxide dismutase (SOD), catalase, demonstrating indirect action as possible antidote (ratio of lead acetate bioinactivation / quercetol $=2.18$ ) [14].

These results have motivated the resumption experiments on laboratory animals to determine other parameters that may be markers of reducing toxic effect [7].

The aim of the present study is to in vivo investigate the influence of quercetol upon urine $\delta$ aminolevulinic acid ( $\Delta-$ ALA) concentration as lead poisoning marker.

\section{Experimental}

The study was conducted on six lots each of 6 mature rats of both sexes, Wistar, weighing specified $(150 \pm 5 \mathrm{~g})$, provided by Cantacuzino Institute, Bucharest, maintained under laboratory bioclimatic conditions and received deionized drinking water ad libidum administered. The animals were treated daily at the same time (9 a.m.), by gavage, with doses of quercetol, lead acetate or deionized water (if applicable) for a period of 12 days.

The researches were conducted at Ovidius University of Constanta. All procedures were performed in accordance with the ECC Directive 86/609/EEC of 24 November 1986 and Ordinance 37 of the Romanian Government from 2 February 2002, regarding research bioethics.

Lots have received the following treatment:

lot 1 - quercetol $0.05 \mathrm{~g} / \mathrm{Kg} /$ body weight;

lot 2 - quercetol $0.1 \mathrm{~g} / \mathrm{kg} /$ body weight;

lot 3 - 20\% lead acetate solution, $0.1 \mathrm{~mL} / \mathrm{kg} /$ body weight;

lot $4-20 \%$ lead acetate solution, $0.1 \mathrm{~mL} / \mathrm{kg} /$ body weight; and quercetol $0.05 \mathrm{~g} / \mathrm{kg} /$ body weight; lot 5 - 20\% lead acetate solution, $0.1 \mathrm{~mL} / \mathrm{kg} /$ body weight; and quercetol $0.1 \mathrm{~g} / \mathrm{kg} /$ body; weight; lot 6 - control, deionized water, $0.1 \mathrm{~mL} / \mathrm{kg}$.

Quercetol was administered p.o. by suspending $0.5 \mathrm{~g}$ and $1 \mathrm{~g}$ of sodium carboxymethylcellulose $1 \%$ mucilage completed to $5 \mathrm{~mL}$ with deionized water and lead acetate was administered as a $20 \%$ solution, $0.1 \mathrm{~mL} / \mathrm{kg}$.

Quercetol x $2 \mathrm{H}_{2} \mathrm{O}$, lead acetate and sodium carboxymethylcellulose come from companies Merck and Roth.

After 11 days of dosing was collected urine from 24 hours to determine the $\Delta$-aminolevulinic acid concentration.

\section{Urine $\delta$-ALA ( $\delta$-aminolevulinic acid) assay}

$\triangle$-ALA is the first intermediate substrate in the synthesis of heme and is neurotoxic, at least in animals [5 - 7, 12 - 14]. Quantification of $\Delta$-ALA can also be achieved by colorimetric methods based on $\delta$-aminolevulinic acid condensation with acetylacetone at $\mathrm{pH}=4.6$, resulting a pyrrole structure which reacts with $\mathrm{p}$-dimethylbenzaldehyde (PABA) in ethhyl acetate medium to form a red compound [15].

Urine $\delta$-aminolevulinic acid concentration is calculated using the formula:

$$
m g \delta-A L A / L \text { urine }=\frac{A P-A P B}{A S} \times 50(1)
$$

where:

$$
\begin{aligned}
& \text { AP - sample absorbance, } \\
& \text { APB - blank absorbance, } \\
& \text { AS - standard absorbance }
\end{aligned}
$$

Colorimetric measurements have been done using a Jasco V-630 spectrophotometer.

Urine normal values for $\delta$-aminolevulinic acid are considered to be in the $0.1-5.7 \mathrm{mg} / \mathrm{L}$ range.

In lead poisoning, excretion of $\delta$ - ALA in the urine increases, and may reach $40 \mathrm{mg} / \mathrm{L}$ [13)].

In order to evaluate the results of statistical calculations, it is necessary to decide whether the differences obtained between analyzed samples are random or real by testing the significance of mean difference $\delta$-aminolevulinic acid concentration by "t" test Student at $\mathrm{p}<0.05$ [11].

To this aim we made the following notations: L2Q2 - quercetol $0.1 \mathrm{~g} / \mathrm{kg} /$ body weight; 
$\mathrm{L} 3 \mathrm{~Pb}-20 \%$ lead acetate solution, $0.1 \mathrm{~mL} / \mathrm{kg}$ / body weight;

$\mathrm{L} 4 \mathrm{Q} 1 \mathrm{~Pb}-20 \%$ lead acetate solution, $0.1 \mathrm{~mL} /$ $\mathrm{kg} /$ body weight; and quercetol $0.05 \mathrm{~g} / \mathrm{kg} /$ body weight;

L5Q2Pb - 20\% le weight; ad acetate solution, $0.1 \mathrm{~mL} / \mathrm{kg} /$ body weight; and quercetol $0.1 \mathrm{~g} / \mathrm{kg} /$ body ;

L6M - control, deionized water, $0.1 \mathrm{~mL} / \mathrm{kg}$.

We analyzed the significance of mean difference $\delta$-aminolevulinic acid concentration as follows: L2Q2-L3Pb, L2Q2-L4Q1Pb, L2Q2L5Q2Pb, L2Q2-L6M, L3Pb-L2Q2, L3Pb-L4Q1Pb, L3Pb-L5Q2Pb, L3Pb-L6M, L4Q1Pb-L5Q2Pb, L4Q1Pb-L6M, L5Q 2Pb-L6M.

\section{Results and Discussions}

Urine $\delta$-aminolevulinic acid concentration variation is given per lot in cumulative table, Table $\mathbf{1}$.
Results are expressed as mean values \pm standard deviation.

Table 1. Urine $\delta$-aminolevulinic acid concentration variation (lots 2-6)

\begin{tabular}{|c|c|}
\hline Lot & $\begin{array}{c}\delta \text {-aminolevulinic acid } \mathrm{mg} / \mathrm{L} \\
(\overline{\mathrm{x}} \pm \text { S.D. })\end{array}$ \\
\hline 2 & $4.39 \pm 2.61$ \\
\hline 3 & $46.63 \pm 9.89$ \\
\hline 4 & $33.18 \pm 7.54$ \\
\hline 5 & $36.48 \pm 11.88$ \\
\hline 6 & $2.72 \pm 0.14$ \\
\hline
\end{tabular}

Tables 2 - 6 present results of significance analysis mean difference $\delta$-aminolevulinic acid concentration determined in urine at the end of the experiment. Significant values of parameter significance analysis are noted where appropriate in bold italics.

Table 2. Influence of quercetol treatment on $\delta$-aminolevulinic acid concentration in lead acetate poisoning. Significance of mean difference by " $\mathrm{t}$ " test student at $\mathrm{p}<0.05$ for L2Q2

\begin{tabular}{|c|c|c|c|c|c|c|c|c|}
\hline Variables & $\begin{array}{c}\text { Mean } \\
\text { (conc. } \mathrm{mg} / \mathrm{L} \text { ) }\end{array}$ & Standard dev. & $\begin{array}{l}\text { Nr. } \\
\text { cases }\end{array}$ & $\begin{array}{c}\text { Mean } \\
\text { dif. }\end{array}$ & $\begin{array}{c}\text { Standard dev. of } \\
\text { dif. }\end{array}$ & $\mathrm{t}$ & $\begin{array}{l}\text { Liberty } \\
\text { grades }\end{array}$ & $\mathrm{p}$ \\
\hline L2Q2 & 4.396 & 3.693 & & & & & & \\
\hline $\mathrm{L} 3 \mathrm{~Pb}$ & 52.146 & 16.074 & 3 & $\begin{array}{c}- \\
47.75 \\
\end{array}$ & 13.724 & -6.02 & 2 & 0.026 \\
\hline L2Q2 & 4.396 & 3.693 & & & & & & \\
\hline $\mathrm{L} 4 \mathrm{Q} 1 \mathrm{~Pb}$ & 37.386 & 12.232 & 3 & $\begin{array}{c}- \\
32.99\end{array}$ & 9.415 & -6.06 & 2 & 0.026 \\
\hline L2Q2 & 4.396 & 3.693 & & & & & & \\
\hline L5Q2Pb & 36.516 & 16.845 & 3 & $\begin{array}{c}- \\
32.12 \\
\end{array}$ & 16.572 & -3.35 & 2 & 0.078 \\
\hline L2Q2 & 4.396 & 3.693 & & & & & & \\
\hline L6M & 2.626 & 0.402 & 3 & 1.77 & 3.988 & 0.768 & 2 & 0.5224 \\
\hline
\end{tabular}


Table 3. Influence of quercetol treatment on $\delta$-aminolevulinic acid concentration in lead acetate poisoning. Significance of mean difference by " $\mathrm{t}$ " test student at $\mathrm{p}<0.05$ for $\mathrm{L} 3 \mathrm{~Pb}$

\begin{tabular}{|l|c|c|c|c|c|c|c|c|}
\hline Variables & $\begin{array}{c}\text { Mean } \\
\text { (conc. } \\
\text { mg/L) }\end{array}$ & $\begin{array}{c}\text { Standard } \\
\text { dev. }\end{array}$ & $\begin{array}{c}\text { Nr. } \\
\text { cases }\end{array}$ & Mean dif. & $\begin{array}{c}\text { Standard } \\
\text { dev. of dif. }\end{array}$ & $\mathrm{t}$ & $\begin{array}{c}\text { Liberty } \\
\text { grades }\end{array}$ & $\mathrm{p}$ \\
\hline $\mathrm{L} 3 \mathrm{~Pb}$ & 52.146 & 16.074 & & & & & & \\
\hline $\mathrm{L} 2 \mathrm{Q} 2$ & 4.396 & 3.69 & 3 & 47.75 & 13.7245 & 6.026 & 2 & $\mathbf{0 . 0 2 6 4}$ \\
\hline $\mathrm{L} 3 \mathrm{~Pb}$ & 46.632 & 17.142 & & & & & & \\
\hline $\mathrm{L} 4 \mathrm{Q} 1 \mathrm{~Pb}$ & 33.182 & 13.056 & 4 & 13.45 & 15.206 & 1.769 & 3 & 0.175 \\
\hline $\mathrm{L} 3 \mathrm{~Pb}$ & 52.146 & 16.074 & & & & & & \\
\hline $\mathrm{L} 5 \mathrm{Q} 2 \mathrm{~Pb}$ & 36.516 & 16.845 & 3 & 15.63 & 29.158 & 0.928 & 2 & 0.451 \\
\hline $\mathrm{L} 3 \mathrm{~Pb}$ & 46.63 & 17.142 & & & & & & \\
\hline $\mathrm{L} 6 \mathrm{M}$ & 2.69 & 0.357 & 4 & 43.935 & 17.236 & 5.098 & 3 & $\mathbf{0 . 0 1 4 5}$ \\
\hline
\end{tabular}

Table 4. Influence of quercetol treatment on $\delta$-aminolevulinic acid concentration in lead acetate poisoning. Significance of mean difference by "t" test student at $\mathrm{p}<0.05$ for L4Q1Pb

\begin{tabular}{|c|c|c|c|c|c|c|c|c|}
\hline Variables & $\begin{array}{c}\text { Mean } \\
\text { (conc. } \\
\mathrm{mg} / \mathrm{L})\end{array}$ & $\begin{array}{c}\text { Standard } \\
\text { dev. }\end{array}$ & $\begin{array}{c}\text { Nr. } \\
\text { cases }\end{array}$ & Mean dif. & $\begin{array}{c}\text { Standard } \\
\text { dev. of dif. }\end{array}$ & $\mathrm{t}$ & $\begin{array}{c}\text { Liberty } \\
\text { grades }\end{array}$ & $\mathrm{p}$ \\
\hline $\mathrm{L} 4 \mathrm{Q} 1 \mathrm{~Pb}$ & 37.386 & 12.232 & & & & & & \\
\hline $\mathrm{L} 2 \mathrm{Q} 2$ & 4.396 & 3.693 & 3 & 32.99 & 9.415 & 6.068 & 2 & $\mathbf{0 . 0 2 6}$ \\
\hline $\mathrm{L} 4 \mathrm{Q} 1 \mathrm{~Pb}$ & 33.182 & 13.056 & & & & & & \\
\hline $\mathrm{L} 3 \mathrm{~Pb}$ & 46.632 & 17.142 & 4 & -14.45 & 15.206 & -1.769 & 3 & 0.175 \\
\hline $\mathrm{L} 4 \mathrm{Q} 1 \mathrm{~Pb}$ & 33.182 & 13.056 & & & & & & \\
\hline $\mathrm{L} 5 \mathrm{Q} 2 \mathrm{~Pb}$ & 36.516 & 16.845 & 3 & 0.87 & 11.945 & 0.126 & 2 & 0.911 \\
\hline $\mathrm{L} 4 \mathrm{P} 1 \mathrm{~Pb}$ & 33.182 & 13.056 & & & & & & \\
\hline $\mathrm{L} 6 \mathrm{M}$ & 2.697 & 0.357 & 4 & 30.485 & 13.394 & 4.551 & 3 & $\mathbf{0 . 0 1 9}$ \\
\hline
\end{tabular}

Table 5. Influence of quercetol treatment on $\delta$-aminolevulinic acid concentration in lead acetate poisoning. Significance of mean difference by " $t$ " test student at $p<0.05$ for L5Q2Pb

\begin{tabular}{|l|c|c|c|c|c|c|c|c|}
\hline Variables & $\begin{array}{c}\text { Mean } \\
\text { (conc. } \\
\mathrm{mg} / \mathrm{L})\end{array}$ & $\begin{array}{c}\text { Standard } \\
\text { dev. }\end{array}$ & $\begin{array}{c}\text { Nr. } \\
\text { cases }\end{array}$ & $\begin{array}{c}\text { Mean } \\
\text { dif. }\end{array}$ & $\begin{array}{c}\text { Standard } \\
\text { dev. of dif. }\end{array}$ & $\begin{array}{c}\text { Liberty } \\
\text { grades }\end{array}$ & $\mathrm{p}$ \\
\hline L5Q2Pb & 36.516 & 16.845 & & & & & & \\
\hline L2Q2 & 4.396 & 3.693 & 3 & 32.12 & 16.572 & 3.356 & 2 & 0.078 \\
\hline L5Q2Pb & 36.516 & 16.845 & & & & & & \\
\hline L3Pb & 52.146 & 16.074 & 3 & -15.63 & 29.158 & -0.928 & 2 & 0.451 \\
\hline L5Q2Pb & 36.516 & 16.845 & & & & & & \\
\hline L4Q1Pb & 37.386 & 12.232 & 3 & -0.87 & 11.945 & -0.126 & 2 & 0.9111 \\
\hline L5Q2Pb & 36.516 & 16.845 & & & & & & 0.076 \\
\hline L6M & 2.626 & 0.402 & 3 & 33.89 & 17.179 & 3.416 & 2 & 0.076 \\
\hline
\end{tabular}


Table 6. Influence of quercetol treatment on $\delta$-aminolevulinic acid concentration in lead acetate poisoning. Significance of mean difference by " $t$ " test student at $\mathrm{p}<0.05$ for L6M

\begin{tabular}{|l|c|c|c|c|c|c|c|c|}
\hline Variables & $\begin{array}{c}\text { Mean } \\
\text { (conc. mg/L) }\end{array}$ & $\begin{array}{c}\text { Standard } \\
\text { dev. }\end{array}$ & $\begin{array}{c}\text { Nr. } \\
\text { cases }\end{array}$ & $\begin{array}{c}\text { Mean } \\
\text { dif. }\end{array}$ & $\begin{array}{c}\text { Standard } \\
\text { dev. of dif. }\end{array}$ & $\mathrm{t}$ & $\begin{array}{c}\text { Liberty } \\
\text { grades }\end{array}$ & $\mathrm{p}$ \\
\hline L6M & 2.626 & 0.402 & & & & & & \\
\hline L2Q2 & 4.396 & 3.693 & 3 & -1.77 & 3.988 & -0.768 & 2 & 0.522 \\
\hline L6M & 2.6975 & 0.357 & & & & & & \\
\hline L3Pb & 46.632 & 17.142 & 4 & -43.935 & 17.236 & -5.098 & 3 & $\mathbf{0 . 0 1 4 5}$ \\
\hline L6M & 2.6975 & 0.357 & & & & & & \\
\hline L4Q1Pb & 33.182 & 13.056 & 4 & -30.845 & 13.394 & -4.551 & 3 & $\mathbf{0 . 0 1 9 8}$ \\
\hline L6M & 2.626 & 0.402 & & & & & & \\
\hline L5Q2Pb & 36.516 & 16.845 & 3 & -33.89 & 17.179 & -3.416 & 2 & 0.0760 \\
\hline
\end{tabular}

Table 7. Influence of quercetol treatment on $\delta$-aminolevulinic acid concentration in lead acetate poisoning. Significance of mean difference by "t" test student at $\mathrm{p}<0.05$ for L4, L3 - L5

\begin{tabular}{|l|c|c|c|c|c|c|c|c|}
\hline Variables & $\begin{array}{c}\text { Mean } \\
\text { (conc. mg/L) }\end{array}$ & $\begin{array}{c}\text { Standard } \\
\text { dev. }\end{array}$ & $\begin{array}{c}\text { Nr. } \\
\text { cases }\end{array}$ & $\begin{array}{c}\text { Mean } \\
\text { dif. }\end{array}$ & $\begin{array}{c}\text { Standard } \\
\text { dev. of dif. }\end{array}$ & $\mathrm{t}$ & $\begin{array}{c}\text { Liberty } \\
\text { grades }\end{array}$ & $\mathrm{p}$ \\
\hline $\mathrm{L} 4 \mathrm{Q} 1 \mathrm{~Pb}$ & 33.182 & 13.056 & 4 & -13.45 & 15.206 & -1.769 & 3 & 0.175 \\
\hline $\mathrm{L} 3 \mathrm{~Pb}$ & 46.632 & 17.142 & 4 & & & & & \\
\hline $\mathrm{L} 5 \mathrm{Q} 2 \mathrm{~Pb}$ & 36.516 & 16.845 & 3 & -15.63 & 29.158 & -0.928 & 2 & 0.451 \\
\hline $\mathrm{L} 3 \mathrm{~Pb}$ & 52.146 & 16.074 & 3 & & & & & \\
\hline
\end{tabular}

Table 7 shows results of significance analysis mean difference of $\delta$-aminolevulinic acid concentration determined in urine at the end of experiment compared to lots $\mathrm{L} 4 \mathrm{Q} 1 \mathrm{~Pb}$ and $\mathrm{L} 5 \mathrm{Q} 2 \mathrm{~Pb}$ $\mathrm{L} 3 \mathrm{~Pb}$.

Statistical analysis of the data presented shows that:

- compared to control group not poisoned (L2Q2 and L6M) the amount of $\delta$-ALA excreted in urine under quercetol influence $(\mathrm{L} 4 \mathrm{Q} 1 \mathrm{~Pb})$ shows statistical significance;

- compared to control group not poisoned (L2Q2) the amount of $\delta$-ALA excreted in urine compared to intoxicated group (L3Pb) shows statistical significance;

- none of any quercetol doses administered (0.05 g / kg b. w. and $0.1 \mathrm{~g} / \mathrm{kg} \mathrm{b}$. w.) did not produce significant changes in the parameters analyzed ( $\delta$-aminolevulinic acid concentration) compared with values of intoxicated and untreated control group (L3Pb);

- difference between means is probably due to sampling fluctuation is not significant and can not be considered to affirm farmacodinamic effect or value;

- $\quad$ reduced growth to eliminate $\delta$-ALA on poisoned and treated groups with quercetol is believed to be due to iron complex formating, reducing hemoglobin synthesis.

\section{Conclusions}

From the results we conclude that hem biosynthesis does not start to grow under quercetol protection.

The obtained data are not relevant statistical since interpretations were performed on non homogeneous groups in number of individuals, the percentage of mortality variability and high levels of standard deviation calculated from each lot. 


\section{References}

*E-mail address: florentinaroncea03@yahoo.com

[1]. F. Roncea, V. Istudor, M. M. Bratu and T Negreanu Pîrjol, Farmacia 40 (2), 55 - 60 (2003) (in Romanian).

[2] F. Roncea, V. Istudor, M. M. Bratu, and T. Negreanu Pîrjol, Magazine of Medicine and Pharmacy 50, Suppliment II, Târgu - Mureş, 271 274 (2004), (in Romanian).

[3]. C. A., Costa., G. C. Trivelato, A. M. P Pinto and E. J. H. Bechara, Clin. Chem. 43 (7), 1196 - 1202 (1997).

[4]. N. Ercal., H. Gurer-Orhan and N. Aykin-Burns, Curr. Top Med. Chem., 1 (6), 124 - 128, (2001).

[5]. S. Kasperczyk, E. Birkner, A. Kasperczyk and J Fiolka - Zalejska, Ann. Agric. Environ. Med., 11, 291 - 296 (2004).

[6]. F. Roncea, Doctoral thesis, The University of Medicine and Pharmacy „Carol Davila”, Bucharest (2007), (in Romanian, abstract in English).

[7]. Goodman Gilman's and Joel. G. - The Pharmacological basis of therapeutics, Raven Press, N.Y. 1996, pp. 1645 - 1666.
[8]. V. A. Voicu - Clinical Toxicology, Albatros Press, Bucharest, 1997, p. 121 - 126 (in Romanian).

[9]. B. Kędzia, E. HołdErna-Kędzia, Herba Polonica, 55 (1), 99 - 108 (2009).

[10]. M. Enoiu, Farmacia 49 (3), 7 - 25 (2001) (in Romanian).

[11]. C. M. Smith, X. Wang., H. Hu and K. Kelsey, Environ. Health Perspect. 103, 248 - 253 (1995).

[12]. *** Romanian Pharmacopoeia, $\mathrm{X}^{\text {th }}$ ed., Medical Press, Bucharest, 2009, p. 1125 - 1154 (in

Romanian).

[13]. M. Cotrău and M. Proca - Clinical toxicology, Medical Press, Bucharest, 1988, p. 103 - 116 (in Romanian).

[14]. G Țarălungă, F. Roncea, V. Istudor, Farmacia, 58 (1), 54 - 61 (2010).

[15]. N. Roşoiu, M. Şerban, Gh. Badiu Laboratory methods and techniques used in biochemistry, vol. II, Clincal biochemistry, Muntenia\&Leda Press, Constantza, 2000, p. 330 333 (in Romanian).

Submitted: May $21^{\text {th }} 2013$ Accepted in revised form: August $7^{\text {th }} 2013$ 